\title{
Can Herbal Medicine Cause Hematoma Enlargement of Hypertensive Intracerebral Hemorrhage within 24 hrs Time Window? A Retrospective Study of 256 Cases from a Single Center in China
}

\author{
Yafa Xu, ${ }^{1,2}$ Jianwen Guo, ${ }^{1}$ Xian Liu, ${ }^{1}$ Juehui Li, ${ }^{1}$ Jing Wang, ${ }^{1}$ and Lingbo Hou ${ }^{1}$ \\ ${ }^{1}$ No. 1 Neurology Department, Guangdong Province Hospital of TCM, The Second Teaching Hospital of Guangzhou University of \\ Traditional Chinese Medicine, 111 Da'de Road, Yuexiu District, Guangzhou, Guangdong Province 510120, China \\ ${ }^{2}$ Neurology Department, Qingyuan Traditional Chinese Medicine Hospital, Qingyuan, Guangdong Province 511500, China \\ Correspondence should be addressed to Jianwen Guo; jianwen_guo@me.com
}

Received 27 May 2014; Accepted 14 October 2014

Academic Editor: Paul Posadzki

Copyright (C) 2015 Yafa Xu et al. This is an open access article distributed under the Creative Commons Attribution License, which permits unrestricted use, distribution, and reproduction in any medium, provided the original work is properly cited.

A retrospective review was performed of consecutive patients presenting with $\mathrm{HICH}$ within 24 hours of ictus presenting between March 2008 and March 2013 who were diagnosed as having HICH by CT scan. Of the 256 patients who matched study inclusion standard, 43 patients hematoma was enlarged (16.8\%). The number of the patients who did not take PBC or RBC herbal medicine, took the PBC herbal medicine, and took RBS herbal medicine was 19 (44.2\%), 2 (4.7\%), and 22 (51.2\%) in hematoma enlargement group and 78 (36.6\%), 26 (12.2\%), and 109 (51.2\%) in nonhematoma enlargement group, individually. There was no significant difference between two groups $(P=0.671)$. PBC and RBS herbal medicine did not increase the incidence of hematoma expansion of ICH within 24 hours after onset of symptom.

\section{Introduction}

Hypertensive intracerebral hemorrhage $(\mathrm{HICH})$ is the most devastating form of stroke. Approximately $40 \%$ of patients with intracerebral hemorrhage die within 30 days, and the majority of survivors are left with severe disability $[1,2]$. Hematoma growth occurs in up to two-third of ICH patients within 24 hours after the onset of symptoms [3]. Furthermore, hemorrhage expansion is an independent determinant of death and disability $[4,5]$. Several reasons may be related to the hematoma enlargement in the early stage of $\mathrm{HICH}$, including high blood pressure, "spot" sign of CT scan, sex, age, time window, and anticoagulation drugs [6]. Herbal medicine of promoting blood circulation (PBC) and removing blood stasis (RBS) are widely used in Chinese hospitals to treat $\mathrm{HICH}$; however, whether this herbal medicine can cause hematoma enlargement is undefined until now [7].

In order to evaluate the safety of PBC and RBS herbal drugs, we designed a retrospective study on the hematoma enlargement in $\mathrm{HICH}$ patients of 256 cases treated with PBC and RBS herbal medicine within 24-hour time window from the symptom onset in Guangdong Province Hospital of Traditional Chinese Medicine.

\section{Material and Methods}

2.1. Materials. A retrospective review was performed of consecutive patients presenting with ICH within 24 hours of ictus presenting between March 2008 and March 2013 who were diagnosed as having hypertensive intracerebral hemorrhage by CT scan. The inclusion criteria also included the following: (1) the patient had hypertensive history; (2) patients got follow-up 24-hour unenhanced CT scan; (3) the patients administrated the herbal medicine within 24 hours from symptom onset of $\mathrm{HICH}$. The exclusion criteria were (1) the time window over $24 \mathrm{hrs}$ from onset to the first CT scan; (2) other reasons causing ICH, such as cerebral tumor, aneurysm, arteriovenous malformation, trauma, anticoagulation drugs, 
and hematological disorder; (3) lack of second CT scan; (4) lack of other important data of the study. Finally, two hundred and fifty-six cases were reviewed.

2.2. Methods. We searched the patients data from the electronic medical record system platform developed by IBM, inc. The searching strategy was "diagnosis=intracerebral hemorrhage" OR “The International Statistical Classification of Diseases and Related Health Problems 10th Revision (ICD10) $[8] "=I 61$, Intracerebral haemorrhage (Excl.: sequelae of intracerebral haemorrhage) (I69.1) OR I61.0 Intracerebral haemorrhage in hemisphere, subcortical (Deep intracerebral haemorrhage) OR I61.1 Intracerebral haemorrhage in hemisphere, cortical (Cerebral lobe haemorrhage, Superficial intracerebral haemorrhage) OR I61.2 (Intracerebral haemorrhage in hemisphere, unspecified) OR I61.3 (Intracerebral haemorrhage in brain stem) OR I61.4 (Intracerebral haemorrhage in cerebellum) OR I61.5 (Intracerebral haemorrhage, intraventricular) OR I61.6 (Intracerebral haemorrhage, multiple localized) OR I61.8 (Other intracerebral haemorrhage) OR I61.9 (Intracerebral haemorrhage, unspecified), "Admission time=March 2008 to March 2013", "SEX=BOTH", "AGE=ALL".

The patients' raw data were recorded in the well-designed case report forms (CRFs) by two researchers, which contained human demography, medical history, personal history, clinical feather, CT scan, laboratory examination, and herbal medicine treatment. The hematoma volume was measured by $\mathrm{ABC} / 2$ Coniglobus formula $[9,10]$. Hemorrhage growth was operationally defined as an increase in the volume of intracerebral hemorrhage of $>33 \%$ as measured by image analysis on the 24-hour CT compared with the baseline CT scan [11].

We defined the herbal medicine as PBC or RBS under the criteria of Chinese Pharmacopoeia of 2010 version. The combined herbal drugs, such as relieving heat and calming liver Yang, decreasing wind and dispersing phlegm, and loosing the bowels, were also under the criteria of Chinese Pharmacopoeia of 2010 version.

Two neuroradiologists analysis on the CT scan data at the work station independently. We divided the patients into hematoma enlargement group and nonhematoma enlargement group. Thus, all the data were analyzed in the statistic software.

2.3. Statistical Analysis. Statistical Product and Service Solutions (SPSS Inc.) 19.0 version was used in our study. Firstly, Univariate analysis was used. $\chi^{2}$ and nonpaired $t$-tests were used to compare patients with and without hemorrhage growth as to the following variables: age, sex, race, current smoking, prior stroke, diabetes, history of hypertension, blood pressure, location of hemorrhage, volume of ICH on baseline CT, time to first CT scan, baseline platelet count, and baseline prothrombin and partial thromboplastin times. The Wilcoxon rank sum test was used to compare the initial GCS score in patients with and without hemorrhage growth. The Wilcoxon rank sum test was also used to compare patients with and without hemorrhage growth as to the change in the GCS score, hematoma enlargement from baseline to 24 hours. Logistic regression was used to investigate possible multiple risk factors and PBC herbal drugs or RBS herbal drugs for growth in hemorrhage volume from baseline to 24 hours. We also analyzed the possible risk factors and PBC and RBS herbal drugs of 3-month outcome followup (mRS 0-1 as independent outcome, mRS 2-6 as dependent outcome) by logistic regression. All statistical tests were two-tailed, and $P \leq 0.05$ was considered significant. Data are presented as mean $\pm \mathrm{SD}$.

\section{Results}

3.1. Patients. Between March 2008 and March 2013, ICH was diagnosed in 901 patients at our hospital. Of these 901 patients, 31 were diagnosed as having bleeding infarction, 43 were diagnosed as having arteriovenous malformation (AVM), 19 were diagnosed as having intracerebral aneurysm, 9 were diagnosed as having cerebral tumor, and 8 were diagnosed as having cerebral trauma. So 791 who were cause by hypertension.

Of the $791 \mathrm{ICH}$ patients, 357 who were admitted after 24 hours of onset were excluded, including 95 patients whose duration was from $24 \mathrm{hrs}$ to $2 \mathrm{ws}, 96$ patients from 2 ws to $6 \mathrm{~ms}$, and 166 patients more than $6 \mathrm{~ms}$. 70 failed to undergo the second CT because of surgery or death. 108 received emergency surgery within 24 hours after first CT scan. Thus, 256 patients, all of whom underwent the first CT within 24 hours of onset and the second CT within 24 hours after first CT scan, were reviewed in this retrospective study.

We also reviewed the patients three months later after entering hospital through telephone or outpatient department visiting. The modified Rankin scale ( $\mathrm{mRS}$ ) was recorded in the followup (see Figure 1).

3.2. Hematoma Growth and the Age. Of the 256 patients who matched study inclusion standard, 43 patients hematoma were enlarged (16.8\%) within $24 \mathrm{hrs}$ from onset. The mean age was $66 \pm 24.00 \mathrm{yrs}$ in hematoma enlargement group and $65.00 \pm 22.00$ yrs in nonhematoma group; they included 173 male patients and 83 female patients.

3.3. PBC and RBS Herbal Medicine Use in Two Groups. The number of the patients who did not take the PBC and RBS herbal medicine was $19(44.2 \%)$ in hematoma enlargement group and $78(36.6 \%)$ in nonhematoma enlargement group. The number of the patients who took the PBC and RBS herbal medicine was $24(55.8 \%)$ in hematoma enlargement group and $135(63.4 \%)$ in nonhematoma enlargement group $(P=$ 0.390). The number of the patients who took the PBC herbal medicine was $2(4.7 \%)$ in hematoma enlargement group and $26(12.2 \%)$ in nonhematoma enlargement group. The number of the patients who took the RBS herbal medicine were 22 (51.2\%) in hematoma enlargement group and 109 (51.2\%) in nonhematoma enlargement group. There was no significant difference between two groups $(P=0.671)$. Thus, $\mathrm{PBC}$ and RBS herbal medicine could not cause hematoma enlargement of $\mathrm{HICH}$ within $24 \mathrm{hrs}$ time window (Figure 2). 


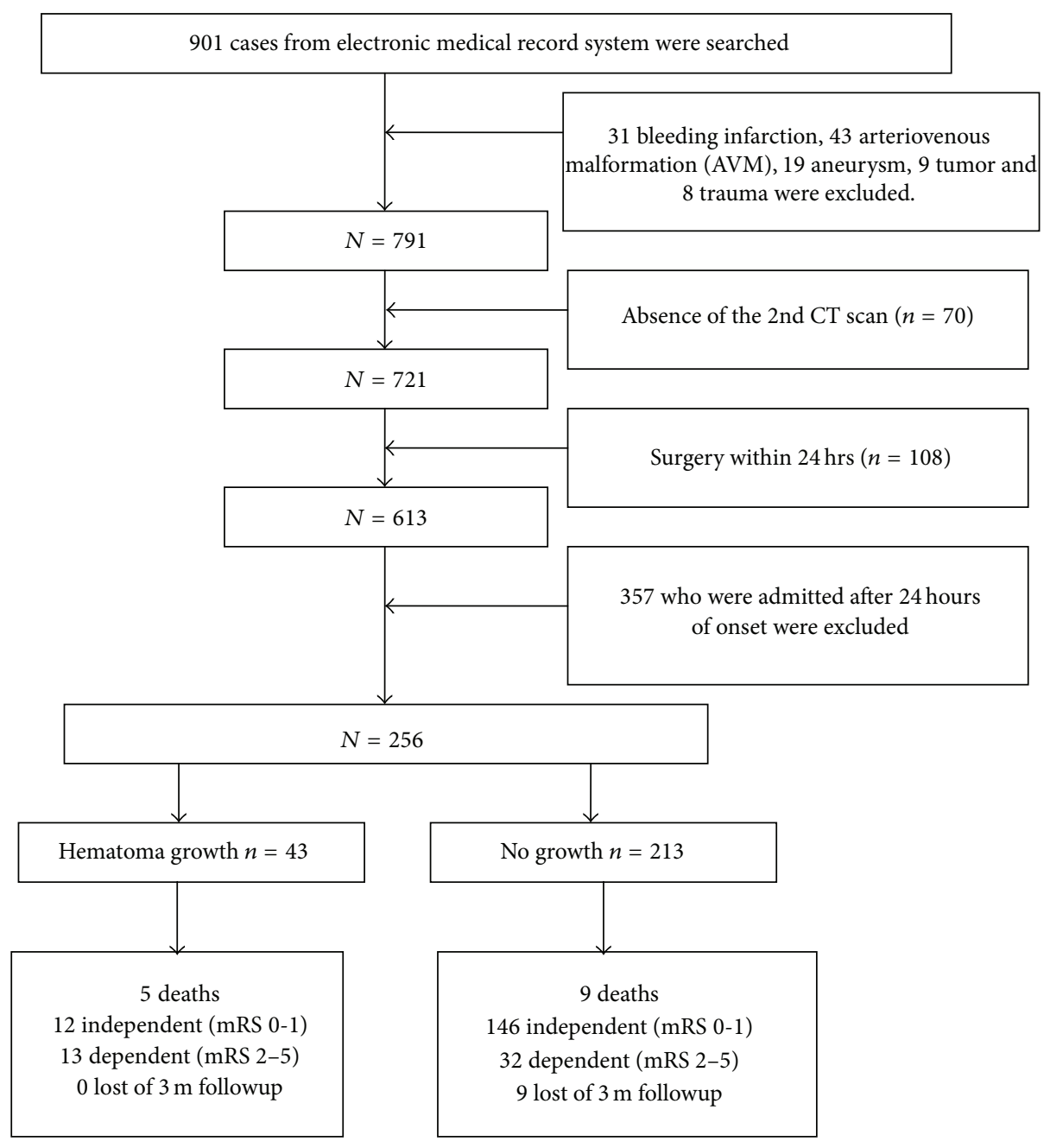

FIgURE 1: Patients recruited chart.

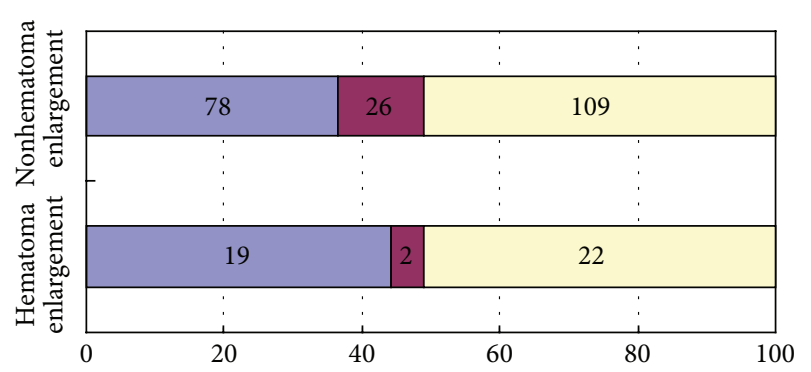

(\%)

Not taken $\square$ RBS

$\mathrm{PBC}$

FIGURE 2: Comparison of PBC and RBS herbal medicine use between two groups.

3.4. Univariate Analysis on the Hematoma Enlargement. We found that patients' sex, baseline GCS, baseline NIHSS, duration from onset to the first CT scan, and aspartate aminotransferase (AST) had significant difference between two groups $(P<0.05)$ (Table 1$)$.

3.5. Multivariate Analysis on the Hematoma Enlargement. Patients' sex, baseline Glasgow coma scale, baseline NIHSS, duration from onset to the first CT scan, aspartate aminotransferase (AST), and PBC and RBS herbal medicine use were an independent variable in the multivariate logistic regression analysis and hematoma growth an outcome variable (dependent variable) (Table 2).

There were two independent factors that can cause hematoma growth. The first one was patient's sex $(P=0.019)$. The second one was duration from onset to the first CT scan, $0-1 \mathrm{hr}(P=0.046), 1-2 \mathrm{hrs}(P=0.041)$. PBC herbal medicine use $(P=0.197)$ or RBS herbal medicine use $(P=0.946)$ was not independent risk fact. On the other hand, the utilization rate of $\mathrm{PBC}$ and RBS herbal medicine was higher in the nonhematoma growth group (63.4\%) than in the hematoma growth group (55.8\%). The coefficient of regression $\beta$ of RBS herbal medicine use was $-1.166, \mathrm{OR}=0.312$. The coefficient of regression $\beta$ of $\mathrm{PBC}$ herbal medicine use was $-0.026, \mathrm{OR}=$ 0.975 . 
TABLE 1: The univariate analysis on the hematoma enlargement (\%).

\begin{tabular}{|c|c|c|c|c|}
\hline Factor & $\begin{array}{c}\text { Hematoma } \\
\text { enlargement }(\%) \\
(n=43)\end{array}$ & $\begin{array}{c}\text { Nonhematoma } \\
\text { enlargement }(\%) \\
(n=213)\end{array}$ & Value & $P$ \\
\hline Age (yrs) & $66.00 \pm 24.00$ & $65.00 \pm 22.00$ & $-0.578^{\triangle}$ & 0.563 \\
\hline Male & $35(81.4)$ & $138(64.8)$ & $4.503^{\diamond}$ & $0.034^{*}$ \\
\hline Hypertension history & $27(62.80)$ & $139(65.30)$ & $0.096^{\diamond}$ & 0.757 \\
\hline DM history & $4(9.30)$ & $25(11.70)$ & $0.038^{\bullet}$ & 0.845 \\
\hline ICH history & $4(9.30)$ & $13(6.10)$ & $0.591^{\diamond}$ & 0.442 \\
\hline \multicolumn{5}{|l|}{ Alcohol intake } \\
\hline Yes & $11(25.6)$ & $44(20.7)$ & & \\
\hline Stopped & $1(2.3)$ & $10(4.7)$ & $0.903^{\diamond}$ & 0.637 \\
\hline No & $31(72.1)$ & $159(74.6)$ & & \\
\hline \multicolumn{5}{|l|}{ Smoking } \\
\hline Yes & $14(32.60)$ & $47(22.10)$ & & \\
\hline Stopped & $3(7.00)$ & $35(16.40)$ & $3.814^{\diamond}$ & 0.149 \\
\hline No & $26(60.50)$ & $131(61.50)$ & & \\
\hline \multicolumn{5}{|l|}{ Duration from onset to the first CT scan (hrs) } \\
\hline $0 \sim 1$ & $6(14.0)$ & $10(4.7)$ & & \\
\hline$>1 \sim 2$ & $9(20.90)$ & $20(9.40)$ & & \\
\hline$>2 \sim 4$ & $13(30.20)$ & $59(27.70)$ & $9.952^{\#}$ & $0.002^{*}$ \\
\hline$>4 \sim 6$ & $3(7.00)$ & $26(12.20)$ & & \\
\hline$>6 \sim 24$ & $12(27.90)$ & $98(46.00)$ & & \\
\hline Baseline systolic blood pressure (mmHg) & $176.42 \pm 31.09$ & $167.82 \pm 25.84$ & $-1.920^{\wedge}$ & 0.056 \\
\hline \multicolumn{5}{|l|}{ Baseline GCS } \\
\hline $3 \sim 7$ & $4(9.30)$ & $4(1.90)$ & & \\
\hline $8 \sim 13$ & $17(39.5)$ & $45(21.1)$ & $12.995^{\#}$ & $0.000^{*}$ \\
\hline $14 \sim 15$ & $22(51.2)$ & $164(77.0)$ & & \\
\hline Baseline NIHSS & $10 \pm 6$ & $5 \pm 7$ & $3.791^{\triangle}$ & $0.000^{*}$ \\
\hline \multicolumn{5}{|l|}{ Hematoma location } \\
\hline Basal ganglion & $19(44.2)$ & $116(54.4)$ & & \\
\hline Thalamus & $4(9.3)$ & $37(17.4)$ & & \\
\hline Lobar & $16(37.2)$ & $46(21.6)$ & $6.667^{\diamond}$ & 0.155 \\
\hline Cerebellar & $1(2.3)$ & $6(2.8)$ & & \\
\hline Brain stem & $3(7.0)$ & $8(3.8)$ & & \\
\hline Intraventricular hemorrhage & $5(11.6)$ & $28(13.1)$ & $0.073^{\diamond}$ & 0.786 \\
\hline \multicolumn{5}{|l|}{ Hematoma volume $(\mathrm{mL})$} \\
\hline$\leq 15$ & $28(65.1)$ & $166(77.9)$ & & \\
\hline$>15 \sim 30$ & $9(20.9)$ & $33(15.5)$ & $3.290^{\#}$ & 0.070 \\
\hline$>30$ & $6(14.0)$ & $14(6.5)$ & & \\
\hline Irregular hematoma & $34(79.1)$ & $139(65.3)$ & $3.115^{\diamond}$ & 0.078 \\
\hline $\operatorname{PLT}\left(10^{9} / \mathrm{L}\right)$ & $203.00 \pm 82.00$ & $209.00 \pm 63.50$ & $-0.768^{\triangle}$ & 0.443 \\
\hline $\mathrm{PT}(\mathrm{s})$ & $11.50 \pm 1.80$ & $12.00 \pm 1.80$ & $-0.270^{\triangle}$ & 0.787 \\
\hline APTT (s) & $30.00 \pm 11.20$ & $30.00 \pm 9.25$ & $-0.026^{\triangle}$ & 0.979 \\
\hline FIB (g/L) & $3.07 \pm 0.63$ & $3.09 \pm 0.71$ & $-0.466^{\triangle}$ & 0.641 \\
\hline ALT (IU/L) & $28.00 \pm 17.00$ & $21.00 \pm 17.00$ & $1.525^{\triangle}$ & 0.127 \\
\hline AST (IU/L) & $29.00 \pm 11.00$ & $24.00 \pm 14.00$ & $1.969^{\triangle}$ & $0.049^{*}$ \\
\hline Urea (mmol/L) & $5.00 \pm 1.44$ & $5.00 \pm 1.72$ & $-0.285^{\triangle}$ & 0.775 \\
\hline Crea $(\mathrm{mmol} / \mathrm{L})$ & $80.00 \pm 28.00$ & $80.00 \pm 23.50$ & $0.388^{\triangle}$ & 0.698 \\
\hline
\end{tabular}


TABle 1: Continued.

\begin{tabular}{|c|c|c|c|c|}
\hline Factor & $\begin{array}{c}\text { Hematoma } \\
\text { enlargement }(\%) \\
(n=43)\end{array}$ & $\begin{array}{l}\text { Nonhematoma } \\
\text { enlargement }(\%) \\
(n=213)\end{array}$ & Value & $P$ \\
\hline \multicolumn{5}{|c|}{ PBC and RBS herbal } \\
\hline Not used & $19(44.2)$ & $78(36.6)$ & & \\
\hline $\mathrm{PBC}$ & $2(4.7)$ & $26(12.2)$ & $0.181^{\#}$ & 0.149 \\
\hline RBS & $22(51.2)$ & 109 (51.2) & & \\
\hline $\mathrm{PBC}$ and $\mathrm{RBS}$ & $24(55.8)$ & $135(63.4)$ & 0.870 & 0.390 \\
\hline Leech & $22(51.2)$ & $109(51.2)$ & 0.00 & 1.00 \\
\hline Leonurus & $24(58.1)$ & $124(58.2)$ & 0.085 & 0.866 \\
\hline Rhizoma & $22(51.2)$ & $109(51.2)$ & 0.00 & 1.00 \\
\hline
\end{tabular}

TABLE 2: Multivariate regression analysis on the independent risk factors of hematoma enlargement in 256 patients.

\begin{tabular}{|c|c|c|c|c|c|}
\hline \multirow{2}{*}{ Independent variable } & \multirow{2}{*}{$\begin{array}{l}\text { Coefficient of } \\
\text { regression }\end{array}$} & \multirow{2}{*}{ OR } & \multicolumn{2}{|c|}{$95 \% \mathrm{CI}$} & \multirow{2}{*}{$P$ value } \\
\hline & & & Lower & Upper & \\
\hline Male patient & 1.066 & 2.903 & 1.189 & 7.086 & $0.019^{*}$ \\
\hline Baseline NIHSS & 0.089 & 1.094 & 0.993 & 1.204 & 0.089 \\
\hline \multicolumn{6}{|l|}{ Baseline GCS (14 15) } \\
\hline Baseline GCS (8 13) & 0.970 & 1.346 & 0.525 & 3.451 & 0.536 \\
\hline Baseline GCS (3 7) & 1.054 & 2.869 & 0.478 & 17.238 & 0.249 \\
\hline \multicolumn{6}{|l|}{ Duration (>6 24 h) } \\
\hline Duration $(>4 \sim 6 \mathrm{~h})$ & 0.106 & 1.112 & 0.273 & 4.524 & 0.883 \\
\hline Duration $(>2 \sim 4 \mathrm{~h})$ & 0.698 & 2.009 & 0.815 & 4.954 & 0.130 \\
\hline Duration (>1 2h) & 1.126 & 3.082 & 1.046 & 9.083 & $0.041^{*}$ \\
\hline Duration $(0 \sim 1 \mathrm{~h})$ & 1.324 & 3.759 & 1.025 & 13.789 & $0.046^{*}$ \\
\hline AST & 0.008 & 1.008 & 0.997 & 1.018 & 0.145 \\
\hline \multicolumn{6}{|l|}{ Not used } \\
\hline RBS & -1.166 & 0.312 & 0.053 & 1.835 & 0.197 \\
\hline $\mathrm{PBC}$ & -0.026 & 0.975 & 0.461 & 2.058 & 0.946 \\
\hline
\end{tabular}

Note: $*$ means $P<0.05$.

3.6. Comparison of Herbal Drugs Combined with PBS and $R B C$. HICH patients were given herbal drugs formula including several mixed herbal drugs besides PBS and RBS drugs, such as relieving heat and calming liver Yang, decreasing wind and dispersing phlegm, and loosing bowls. We analyse the effect as in Table 3.

The results showed that there was no significant difference between two groups combined with the above three types of herbal drugs (all $P>0.05$ ).

3.7. Multivariate Analysis on the 3-Month Outcome Followup $(m R S)$. Patients' sex, baseline Glasgow coma scale, baseline NIHSS, duration from onset to the first CT scan, aspartate aminotransferase (AST), PBC and RBS herbal medicine use, and hematoma growth were an independent variable in the multivariate logistic regression analysis and $\mathrm{mRS}$ a dependent variable. We defined mRS $0-1$ as independent outcome and mRS 2-6 as dependent outcome.
The results showed that baseline NIHSS and hematoma growth were the independent risk factors of outcome of threemonth followup (see Table 4).

There were two independent factors that affect the 3month outcome. The first one was baseline NIHSS $(P=$ $0.000)$. The second one was hematoma growth $(P=0.003)$. $\mathrm{PBC}$ and RBS herbal medicine use was not independent risk factor $(P=0.651)$.

\section{Discussion}

The safety of the herbal medicine administration became more and more critical since aristolochic acids were reported to cause renal injury in 1993 [12, 13].

It is long history that $\mathrm{PBC}$ and RBS herbal medicine were used in China to treat $\mathrm{HICH}$. A multicenter, prospective clinical trial showed PBC and RBS herbal medicine can reduce the death and also improve the neurological function [14]. Metaanalysis showed that $\mathrm{PBC}$ and RBS herbal medicine seems 
TABLE 3: Herbal drugs combined with PBS and RBC between two groups $(n, \%)$.

\begin{tabular}{|c|c|c|c|c|}
\hline Combined herbal drugs & $\begin{array}{c}\text { PBS and RBC used in } \\
\text { hematoma enlargement } \\
(n, \%) \\
(n=24)\end{array}$ & $\begin{array}{c}\text { PBS and RBC used in } \\
\text { nonhematoma } \\
\text { enlargement }(n, \%) \\
(n=135)\end{array}$ & Value & $P$ \\
\hline $\begin{array}{l}\text { Relieving heat and calming } \\
\text { liver Yang }\end{array}$ & $21(87.5)$ & $116(85.9 \%)$ & 0.042 & 0.837 \\
\hline $\begin{array}{l}\text { Decreasing wind and } \\
\text { dispersing phlegm }\end{array}$ & $14(58.3 \%)$ & $104(77.0 \%)$ & 3.725 & 0.054 \\
\hline Loosing bowls & $19(79.2 \%)$ & $101(74.8 \%)$ & 0.208 & 0.648 \\
\hline
\end{tabular}

TABLE 4: Multivariate regression analysis on the independent risk factors of 3-month outcome in 247 patients.

\begin{tabular}{|c|c|c|c|c|c|}
\hline \multirow{2}{*}{ Independent variable } & \multirow{2}{*}{ Coefficient of regression } & \multirow{2}{*}{ OR } & \multicolumn{2}{|c|}{$95 \%$ CI } & \multirow{2}{*}{$P$ value } \\
\hline & & & Lower & Upper & \\
\hline Sex & 0.293 & 1.341 & 0.630 & 2.852 & 0.447 \\
\hline Baseline NIHSS & -0.397 & 0.672 & 0.598 & 0.755 & 0.000 \\
\hline Baseline GCS & 0.211 & 1.234 & 0.565 & 2.697 & 0.598 \\
\hline Duration from onset & 0.022 & 1.023 & 0.776 & 1.347 & 0.874 \\
\hline AST & -0.006 & 0.994 & 0.980 & 1.009 & 0.439 \\
\hline $\mathrm{PBC}$ and RBS used & 0.164 & 1.178 & 0.580 & 2.392 & 0.651 \\
\hline Hematoma growth & -1.482 & 0.227 & 0.085 & 0.609 & 0.003 \\
\hline
\end{tabular}

effective to treat $\mathrm{HICH}[15]$. PBC and RBS were also adopted by textbook and guideline in treating $\mathrm{HICH}[16]$.

However, safety data about hematoma enlargement were also reported. Bin and Jian declared that danshen injection and mailuoning injection (one of the PBC and RBS herbal medicine) could induce the uncontrolled bleeding [17]. Leech prevents not only fibrinogen clotting but also other thrombin-catalyzed hemostatic reactions such as the activation of clotting factors V, VIII, and XIII and the thrombin-induced platelet activation [18]. Other researchers advocated that PBC and RBC herbal medicine should be used with few side effects in the clinical application because they added to some other stopping bleeding herbal medicine to make the prescription balance [19]. So it is necessary that we perform this study on the safety of treating $\mathrm{HICH}$ with PBC and RBS herbal medicine.

In this study, forty-three (16.8\%) of the 256 patients demonstrated enlargement of the hematoma after the first CT scan. The growth rate was near the rate $14.3 \%$ (60/419) of Fujii et al.s report [20]. In this study, 159 patients were administrated PBC and RBS herbal medicine prescription within 24-hour time window, including 24 patients in hematoma enlargement group and 135 patients in no hematoma group, which were not significantly different $(P>0.05)$.

Hematoma enlargement in $\mathrm{HICH}$ has significant associations with the duration of time since onset of neurological symptoms, the shape and volume of the bleeding, the initials deep coma degree, the presence of liver dysfunction, and male patients $[20,21]$. In this study, the univariate analysis showed that duration, baseline GCS and NIHSS, the liver dysfunction (AST), and male patients were the risk factor of hematoma expansion, while the shape and size of the hematoma were not significant because the sample was too small.
There were only two risk factors that induced hematoma growth according to the multivariate analysis, male patients and duration of onset since ICH symptoms. Hematoma enlargement was the independent risk factor of outcome of three-month followup; the result was similar as Seiji Kazui's study [22]. PBC and RBS herbal drug was not the independent risk factor of hematoma growth of the outcome of three-month followup.

Some herbal drugs have strong PBC and RBS function; for example, leech caused rebleeding [21]. Some others have twoway adjustment pharmacological effect; for example, Radix notoginseng can not only $\mathrm{PBC}$ but also stopping bleeding. In our opinion, the prescription of Chinese medicine to learn is through reasonable compatibility other than a single drug, to eliminate this rebleeding risk.

PBC and RBS herbal medicine was administrated within the time window that was uncertain. A majority (83\%) of patients with hematoma enlargement underwent the initial CT scan within 6 hours of onset; Enlargement after 24 hours of onset seems extremely rare [22]. So some neurologists showed their opinion that $\mathrm{PBC}$ and RBS herbal medicine should be used after $24 \mathrm{hrs}$ of onset in order to prevent rebleeding risk [23]. Others supported that PBC and RBS herbal medicine should be administrated as sooner as possible [24]. Guo and his colleagues' study did not show deterioration of condition of the AICH patients who were treated with herbal compound within 6 hrs time window from onset [25].

$\mathrm{PBC}$ and RBS herbal medicine should be combined with other drugs correctly in the Traditional Chinese Medicine Formula in order to avoid the rebleeding risk, as recorded in the Chinese Pharmacopoeia of 2010 version [26]. In this study, the treatment of acute cerebral hemorrhage frequently 
used herbal medicine by turns as follows: Leonurus japonicus Houtt. (148 cases), Rhizoma Polygoni Cuspidati (131 cases), leech (131 cases), Radix Achyranthis Bidentatae (83 cases), Ligusticum chuanxiong Hort. (21 cases), Radix Salviae Miltiorrhizae (18 cases), Radix et Rhizoma Rhei Palmati (6 cases), Cortex moutan (15 cases), peach seed (13 cases), Radix Curcumae Wenyujin (12 cases), Radix notoginseng (6 cases), Carthamus tinctorius L. (5 cases), Caulis Spatholobi (4 cases), and Rhizoma corydalis ( 2 cases). They were used in balance between two groups $(P>0.05)$.

In this retrospective study, 159 patients (24 in hematoma growth group and 135 in no hematoma growth group) were given $\mathrm{PBC}$ and RBS herbal medicine prescription, which was also combined with other herbal drugs (showed as Table 4). PBC and RBS drugs reasonable compatibility with other herbs perhaps reduced the risk of hematoma expansion caused by single PBC and RBS herb. This result undoubtedly is instructive for further clinical application.

This is the first retrospective study of the hematoma growth on the early $\mathrm{HICH}$ treated with traditional Chinese medicine since now. Though the results showed it seems safe, the retrospective study has many limitations. Firstly, there are too many herbals medication to control the quality of the clinical study. Secondly, a lot of patients were excluded because of important data absent, for example, their second CT scan data. Thirdly, some scales, including NIHSS and GCS, had recall bias from raw medical records.

In order to make up for these limitations, we have designed a prospective, 13 hospitals, randomized, placebo control clinical trial (clinicaltrials.gov: NCT01918722) to confirm if PBC and RBS herbal medicine induces the incidence of hematoma enlargement of AICH patient within the $6 \mathrm{hrs}$ time window from onset. 62 cases have been recruited since February 2014 and all 300 patients will be completed in December 2015.

\section{Conflict of Interests}

The authors declare that there is no conflict of interests regarding the publication of this paper.

\section{Acknowledgment}

The authors thank Professor Zehuai Wen because he directed the statistics of the data. He is the director of Key Unit of Methodology in Clinical Research of Guangdong Province Hospital of Traditional Chinese Medicine. The authors also thank China National Clinical Study Base Project of Traditional Chinese Medicine, sponsored by State Administration of Traditional Chinese Medicine of the People's Republic of China (Grant no. JDZX2012074).

\section{References}

[1] A. I. Qureshi, A. D. Mendelow, and D. F. Hanley, "Intracerebral haemorrhage," The Lancet, vol. 373, no. 9675, pp. 1632-1644, 2009.

[2] C. J. van Asch, M. J. Luitse, G. J. Rinkel, I. van der Tweel, A. Algra, and C. J. Klijn, "Incidence, case fatality, and functional outcome of intracerebral haemorrhage over time, according to age, sex, and ethnic origin: a systematic review and metaanalysis," The Lancet Neurology, vol. 9, no. 2, pp. 167-176, 2010.

[3] J. K. Lim, H. S. Hwang, B. M. Cho et al., "Multivariate analysis of risk factors of hematoma expansion in spontaneous intracerebral hemorrhage," Surgical Neurology, vol. 69, no. 1, pp. 40-45, 2008.

[4] S. M. Davis, J. Broderick, M. Hennerici et al., "Hematoma growth is a determinant of mortality and poor outcome after intracerebral hemorrhage," Neurology, vol. 66, no. 8, pp. 11751181, 2006.

[5] G.-Q. Wang, S.-Q. Li, Y.-H. Huang et al., "Can minimally invasive puncture and drainage for hypertensive spontaneous Basal Ganglia intracerebral hemorrhage improve patient outcome: a prospective non-randomized comparative study," Military Medical Research, vol. 1, article 10, 2014.

[6] J. C. Hemphill III, D. C. Bonovich, L. Besmertis, G. T. Manley, and S. C. Johnston, "The ICH score: a simple, reliable grading scale for intracerebral hemorrhage," Stroke, vol. 32, no. 4, pp. 891-896, 2001.

[7] G. Jian-wen and L. Ming-jie, "Meta-analysis of acute intracerebral hemorrhage treated with traditional Chinese medicine or/and composition of promoting blood circulation and removing blood stasis," Journal of China-Japan Friendship Hospital, vol. 15, no. 5, pp. 283-286, 2001.

[8] ICD-10 Version, 2010, http://apps.who.int/classifications/icd10/ browse/2010/en.

[9] R. U. Kothari, T. Brott, J. P. Broderick et al., "The ABCs of measuring intracerebral hemorrhage volumes," Stroke, vol. 27, no. 8, pp. 1304-1305, 1996.

[10] J. M. Gebel, C. A. Sila, M. A. Sloan et al., "Comparison of the $\mathrm{ABC} / 2$ estimation technique to computer-assisted volumetric analysis of intraparenchymal and subdural hematomas complicating the GUSTO-1 trial," Stroke, vol. 29, no. 9, pp. 1799-1801, 1998.

[11] T. Brott, J. Broderick, R. Kothari et al., "Early hemorrhage growth in patients with intracerebral hemorrhage," Stroke, vol. 28, no. 1, pp. 1-5, 1997.

[12] J.-L. Vanherweghem, M. Depierreux, C. Tielemans et al., "Rapidly progressive interstitial renal fibrosis in young women: association with slimming regimen including Chinese herbs," The Lancet, vol. 341, no. 8842, pp. 387-391, 1993.

[13] M. Jadoul, J. F. de Plaen, J. P. Cosyns, and C. van Ypersele de Strihou, "Adverse effects from traditional Chinese medicine," The Lancet, vol. 341, no. 8849, pp. 892-893, 1993.

[14] P.-X. Huang, Y. Huang, and M. Lu, "Treatment of acute intracerebral hemorrhage with a comprehensive protocol of integrated Chinese and Western medicine," Zhongguo Zhong Xi Yi Jie He Za Zhi, vol. 26, no. 7, pp. 590-593, 2006.

[15] J. Guo and M. Liu, "Meta-analysis of acute intracerebral hemorrhage treated with traditional Chinese medicine or/and composition of promoting blood circulation and removing blood stasis," Journal of China-Japan Friendship Hospital, vol. 15, no. 5, pp. 283-286, 2001.

[16] W. Mian-hua and W. Xin-yue, Traditional Chinese Internal Medicine, China Press of Traditional Chinese Medicine, 3rd edition, 2000.

[17] L. Bin and L. Jian, "Clinical observation of early use promoting blood circulation and removing blood stasis herbal injection to treat acute intracerebral hemorrhage," Shandong Journal of Traditional Chinese Medicine, vol. 19, no. 8, pp. 461-462, 2000. 
[18] F. Markwardt, "The development of hirudin as an antithrombotic drug," Thrombosis Research, vol. 74, no. 1, pp. 1-23, 1994, Review.

[19] J. W. Guo, Y. F. Cai, and B. X. Du, “Thinking and challenge induced by the hypothesis of breaking stagnant and eliminating blood stasis in Treating acute cerebral hemorrhage by rF VII a," Zhongguo Zhong Xi Yi Jie He Za Zhi, vol. 25, no. 11, pp. 10271029, 2005 (Chinese).

[20] Y. Fujii, R. Tanaka, S. Takeuchi, T. Koike, T. Minakawa, and O. Sasaki, "Hematoma enlargement in spontaneous intracerebral hemorrhage," Journal of Neurosurgery, vol. 80, no. 1, pp. 51-57, 1994.

[21] L. Liu, J.-A. Duan, Y. Tang et al., “Taoren-Honghua herb pair and its main components promoting blood circulation through influencing on hemorheology, plasma coagulation and platelet aggregation," Journal of Ethnopharmacology, vol. 139, no. 2, pp. 381-387, 2012.

[22] S. Kazui, H. Naritomi, H. Yamamoto, T. Sawada, and T. Yamaguchi, "Enlargement of spontaneous intracerebral hemorrhage: incidence and time course," Stroke, vol. 27, no. 10, pp. 1783-1787, 1996.

[23] Y. Wanzhng, Z. Zhilan, Z. Ming et al., "Once again on bleeding after cerebral hemorrhage and the time window for therapy of "promoting blood circulation and removing blood stasis"', Chinese Journal of Integrative Medicine on Cardio-/Cerebrovascular Disease, vol. 11, pp. 662-665, 2004.

[24] W. Li, "Discussion of early use promoting blood circulation and removing blood stasis herbal medicine," Chinese Journal of Basic Medicine in Traditional Chinese Medicine, vol. 5, pp. 58-59, 2003.

[25] J.-W. Guo, Y.-C. He, and S.-H. Chen, "Clinical study on the therapeutic time window of acute intracerebral hemorrhage treated with herbal composition of Zhongfengxingnao taken orally," Chinese Journal of Cerebrovascular Diseases, vol. 2, no. 6, pp. 255-259, 2005.

[26] Chinese Pharmacopoeia Commission, The Pharmacopoeia of the People's Republic of China, vol. 1, Chemical Industry Press, Beijing, China, 2010. 


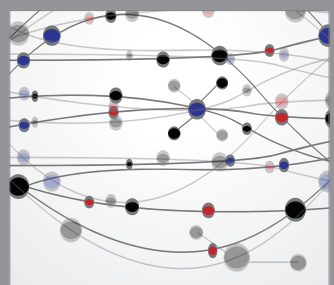

The Scientific World Journal
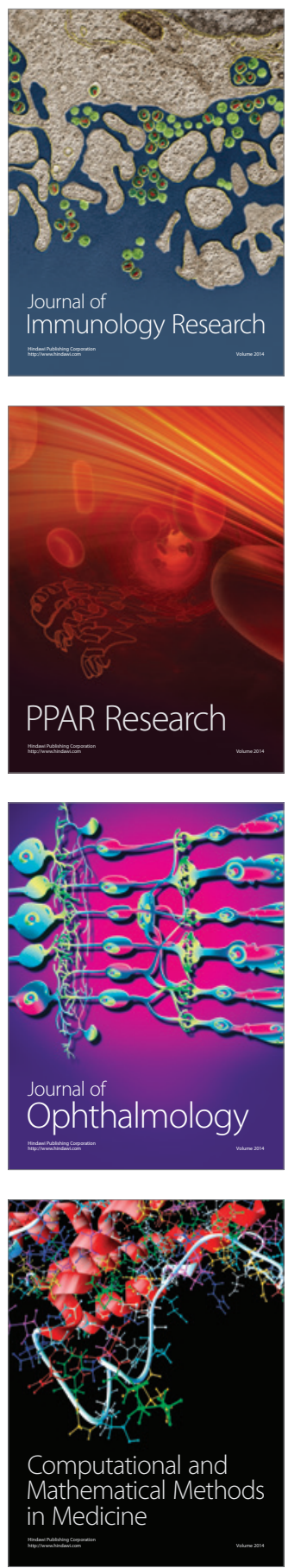

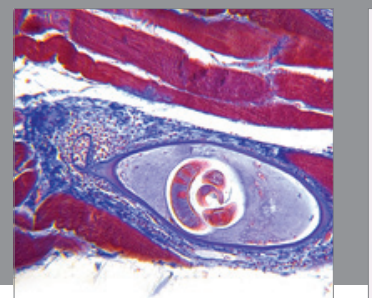

Gastroenterology

Research and Practice
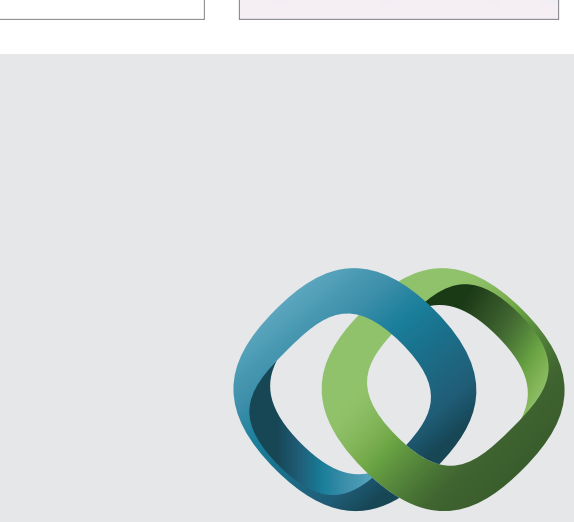

\section{Hindawi}

Submit your manuscripts at

http://www.hindawi.com
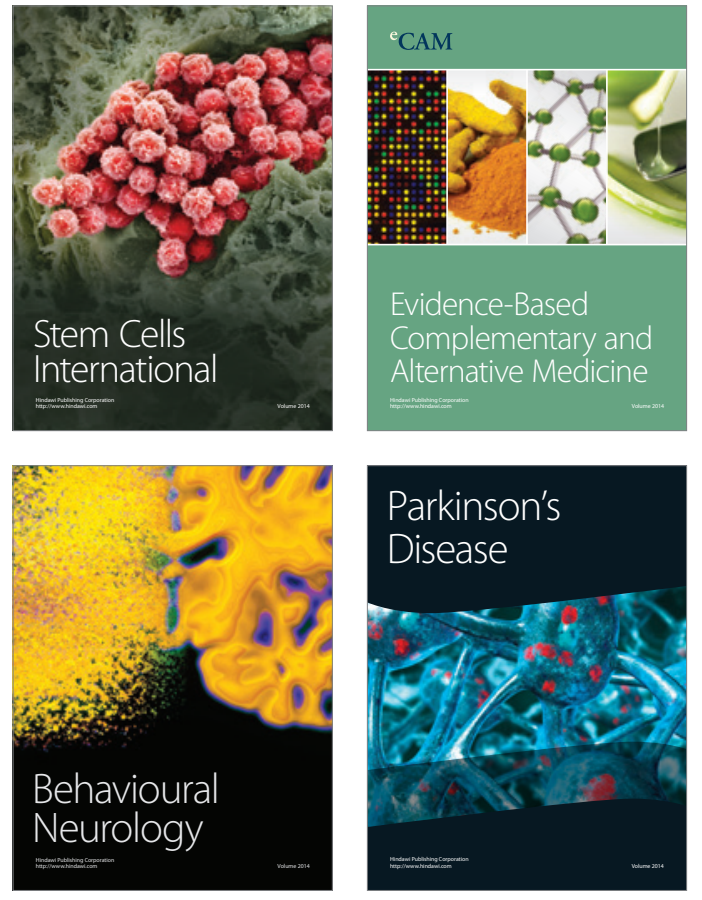
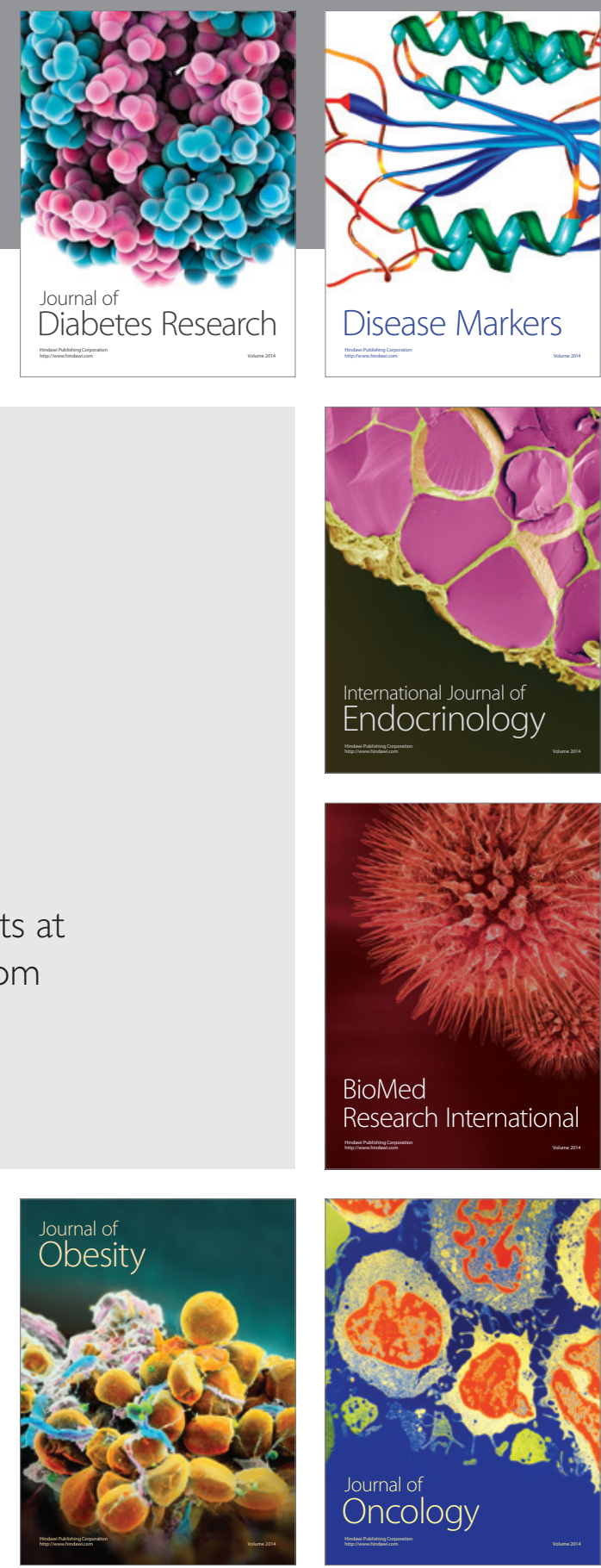

Disease Markers
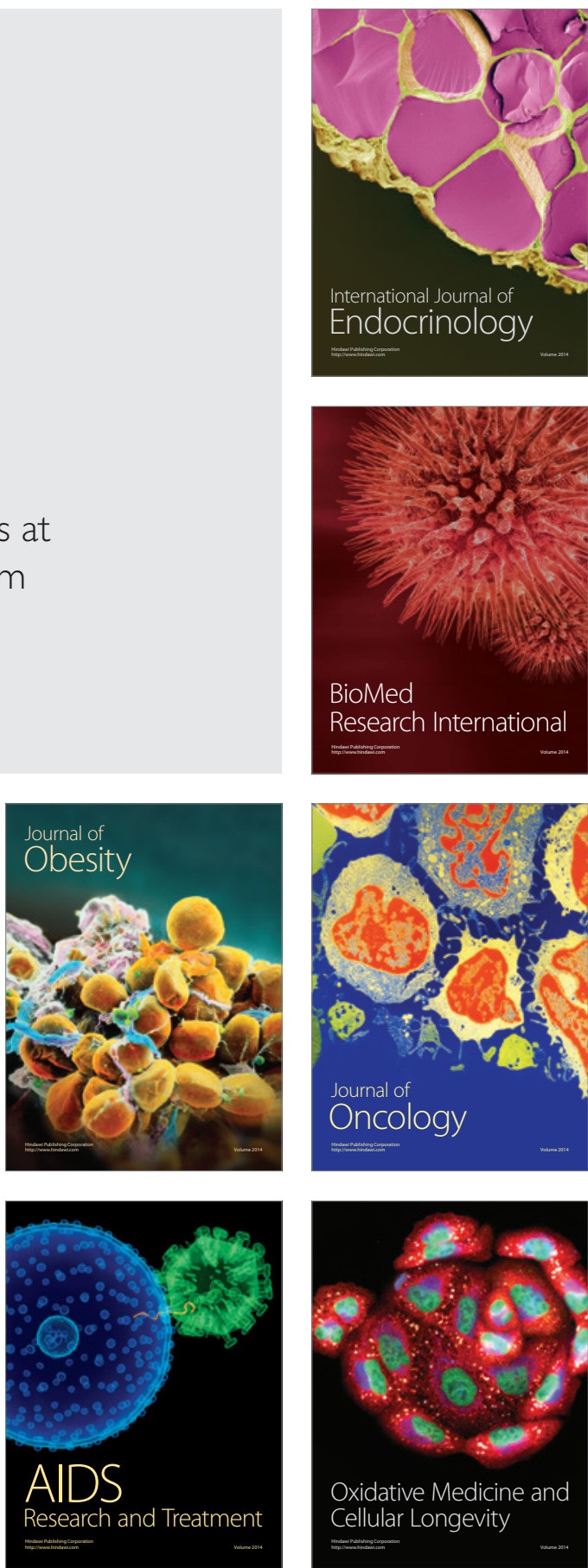